\title{
Proline-based phosphoramidite reagents for the reductive ligation of $S$-nitrosothiols
}

\author{
Chung-Min Park $^{1}$, Tyler D Biggs ${ }^{1}$ and Ming Xian \\ S-Nitrosothiols (RSNOs) have many biological implications but are rarely used in organic synthesis. In this work we report the \\ development of proline-based phosphoramidite substrates that can effectively convert RSNOs to proline-based sulfenamides \\ through a reductive ligation process. A unique property of this method is that the phosphine oxide moiety on the ligation \\ products can be readily removed under acidic conditions. In conjugation with the facile preparation of RSNOs from the \\ corresponding thiols (RSHs), this method provides a new way to prepare proline-based sulfenamides from simple thiol starting \\ materials.
}

The Journal of Antibiotics (2016) 69, 313-318; doi:10.1038/ja.2015.144; published online 13 January 2016

\section{INTRODUCTION}

Sulfur-nitrogen (-S-N-) linkages are unique chemical moieties. These structures often show interesting bioactivities (Figure 1). For example, sulfonylureas are a class of important herbicides. ${ }^{1,2}$ Sulfanilamides have been used as common drugs for infections. ${ }^{3,4} \mathrm{~N}$-Thiolated $\beta$-lactams are found to have antibacterial, antifungal and anticancer effects. ${ }^{5-12}$ Sulfenamides exhibit antimicrobial activities against various infectious pathogens. ${ }^{13}$ Proline-based sulfonamides have been recognized as potential antiproliferative and anti-infective agents. ${ }^{14-16}$ Because of these activities, the preparation of these molecules, especially the formation of the -S-N- linkages, has become an active area in organic synthesis. So far, many methods have been developed for the construction of the S-N bonds, including: (1) sulfenylation between sulfenyl chlorides and amines, ${ }^{17,18}$ (2) amination of thiols with $\mathrm{N}$-halo compounds or amines in the presence of oxidizing reagents to give unsubstituted and $N$-substituted sulfenamides, ${ }^{19}$ (3) the treatment of disulfides with ammonia or amines in the presence of silver or mercuric salts in alkaline medium, ${ }^{20,21}$ (4) sulfenylation of primary and secondary amines with the esters of sulfenic acids, ${ }^{22}$ (5) derivations of $\mathrm{N}$-chlorothiocompounds, ${ }^{23,24}$ (6) the [2,3]-sigmatropic rearrangement of S-allylsulfynimines ${ }^{25}$ (7) reductive cleavage of sulfinimidic acids in the presence of thiophenols, ${ }^{26}$ (8) electrolysis of 2-mercaptobenzothiazole-amine or bisbenzothiazol-2-yldisulfideamine $^{27,28}$ and (9) the preparation of sulfin- or sulfon-amides by the oxidation of sulfenamides in the presence of the oxidants. ${ }^{29,30}$

$\mathrm{S}$-Nitrosothiols (RSNOs) are a group of $-\mathrm{S}-\mathrm{N}$ - bond containing molecules. The formation of $S$-nitrosothiols in biological systems is an important post-translational modification elicited by nitric oxide. ${ }^{31-33}$ RSNOs include protein-based adducts (through cysteine residues) and small molecules (such as $S$-nitrosoglutathione and $S$-nitrosocysteine).
Small organic thiols (RSHs) can also be easily converted to the corresponding RSNOs under mild nitrosation conditions. Normally, RSNOs are unstable species that makes their detection in biological systems very challenging. Moreover, the instability of RSNOs makes these compounds unattractive for synthetic chemists. As a result, the application of RSNOs in synthesis has been rarely reported. Interestingly, in our recent work on RSNO bioorthogonal reactions (aiming at the development of novel detection methods for protein RSNO formation), ${ }^{34-41}$ we have discovered some unusual reactivity and properties of RSNOs. In our opinion, RSNOs are powerful synthons that can be used to introduce $\mathrm{S}, \mathrm{N}$ and/or $\mathrm{O}$ atoms into molecular structures. Herein, we wish to report a method to prepare prolinebased sulfenamides from readily available RSH substrates via RSNO intermediates. The novel reactions and synthetic strategies introduced in this article could be applied for an effective synthesis of bioactive molecules including a proline moiety.

\section{RESULTS AND DISCUSSION}

In our previous work, we discovered that RSNOs and triarylphosphines ( 2 equivalents) can rapidly react to generate reactive thioazaylide intermediates in high yields under mild conditions. ${ }^{34}$ Thioazaylides are potent nucleophilic species. Upon manipulating the electrophilic groups attached to the phosphine reagents, thioazaylides can be trapped as stable products. For example, as shown in Scheme 1, when an ortho-ester group was attached to the phosphine reagent (compound 3 ), the reactive thioazaylide 4 could be trapped via an intramolecular acyl transfer to afford sulfenamide $5 .{ }^{35-37}$ This reductive ligation process provides a way for capturing unstable biological RSNO as stable and detectable conjugates. In our opinion, this reaction, in conjunction with easy RSNO formation from RSH, would be also useful for the synthesis of sulfenamide derivatives from 

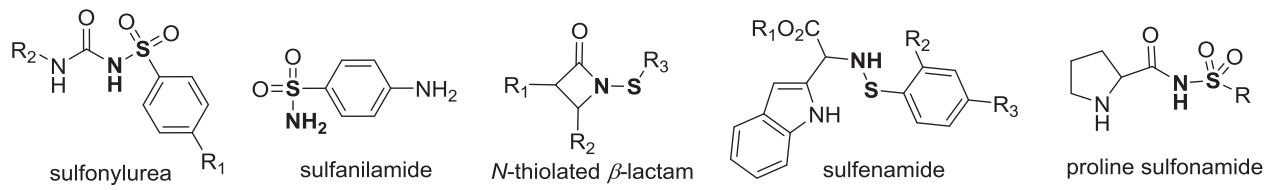

Figure 1 Representative molecules having sulfur-nitrogen (-S-N-) linkages.
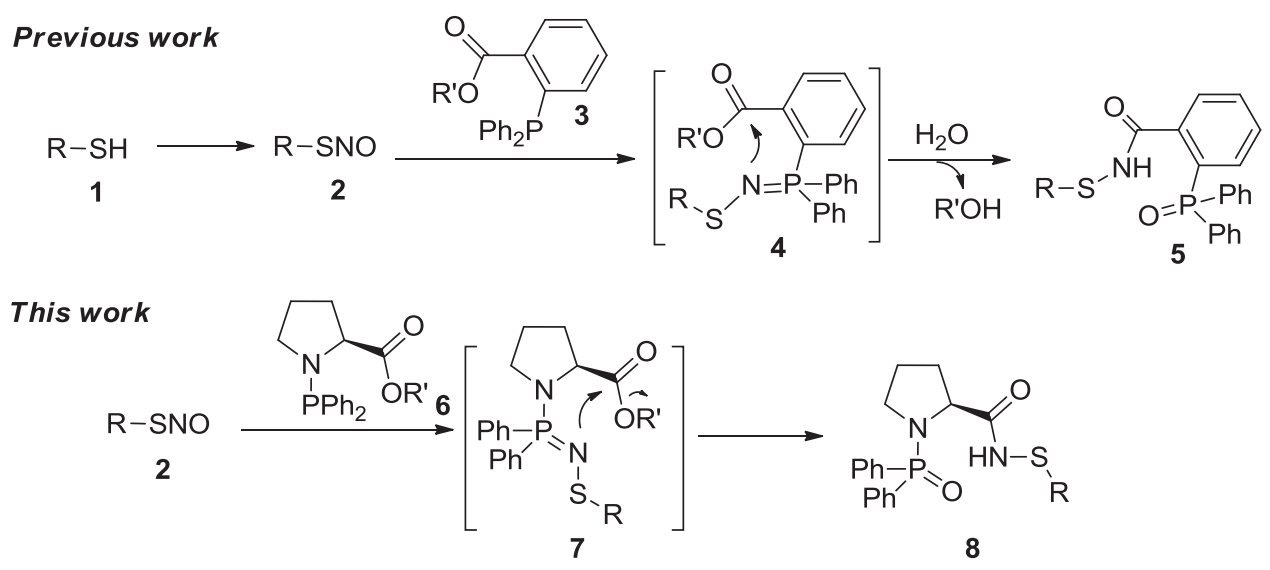

Scheme 1 Reductive ligations of S-nitrosothiols.

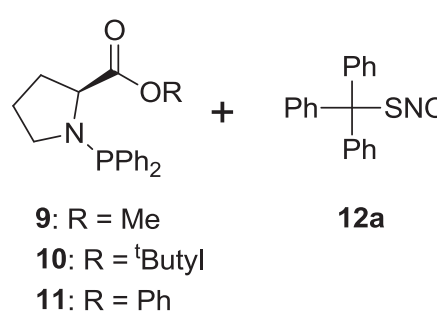

Scheme 2 Reactions between proline-based phosphoramidites and TrSNO.

simple RSH starting materials. However, the use of phosphine substrates like $\mathbf{3}$ would lead to products like $\mathbf{5}$ that contain an unnecessary triphenylphosphine oxide moiety. The removal of this bulky group from the final products would require harsh conditions. Therefore, seeking phosphine substrates that can undergo the reductive ligation while leading to a readily removable phosphine oxide moiety from the products is desirable. With this idea in mind, we proposed that proline-based phosphoramidites like 6 would be suitable substrates. As shown in Scheme 1, the reaction between RSNO and $\mathbf{6}$ should still follow the reductive ligation process. The resultant phosphoramidate moiety can be considered as a $\mathrm{NH}$-protecting group and removable under acidic condition. As such, proline-linked sulfenamides could be prepared from simple RSH and phosphoramidite 6.

To test this idea, three proline-based phosphoramidites (Scheme 2, 9-11) were prepared. These substrates were treated with TrSNO (12a) to explore their reactivity. The selection of TrSNO as the RSNO model compound was because of its remarkable stability and ease of synthesis. We found the characteristic green color of TrSNO disappeared immediately when treated with all three phosphoramidites, suggesting the formation of thioazaylides was fast. For 9 and 10, the reactions stopped at this stage as no desired ligation product was obtained even when the reaction time was extended to $24 \mathrm{~h}$. With 11, however, we obtained the desired ligation product 16a. Apparently, this can be explained by the factor that phenyl ester is a better leaving group than the methyl and $t$-butyl esters. We tested a series of different solvents for this reaction. The best was found to be a mixture of $\mathrm{THF} /$ phosphate buffer ( $\mathrm{pH} 7.4,20 \mathrm{~mm}, 3: 1$ ) that gave 16a in $84 \%$ yield.

As phosphoramidite $\mathbf{1 1}$ was found to be the most reactive substrate for this ligation, it was applied to other RSNO substrates to test the generality of the reaction. A series of primary, secondary and tertiary RSNOs were freshly prepared from RSH and then used in this study without any purification. The results were summarized in Table 1. In all cases, the desired ligation products were obtained. For relatively stable tertiary RSNO substrates, the corresponding ligation products were isolated in modest to high yields (entries 1-4). Secondary and primary RSNO substrates gave slightly lower yields (entries 5-9). Interestingly, the purification of secondary RSNO products (entries 5 and 6) was found to be very difficult because they overlapped with the 
Table 1 Summary of the reactions between 11 and S-nitrosothiols (RSNOs)

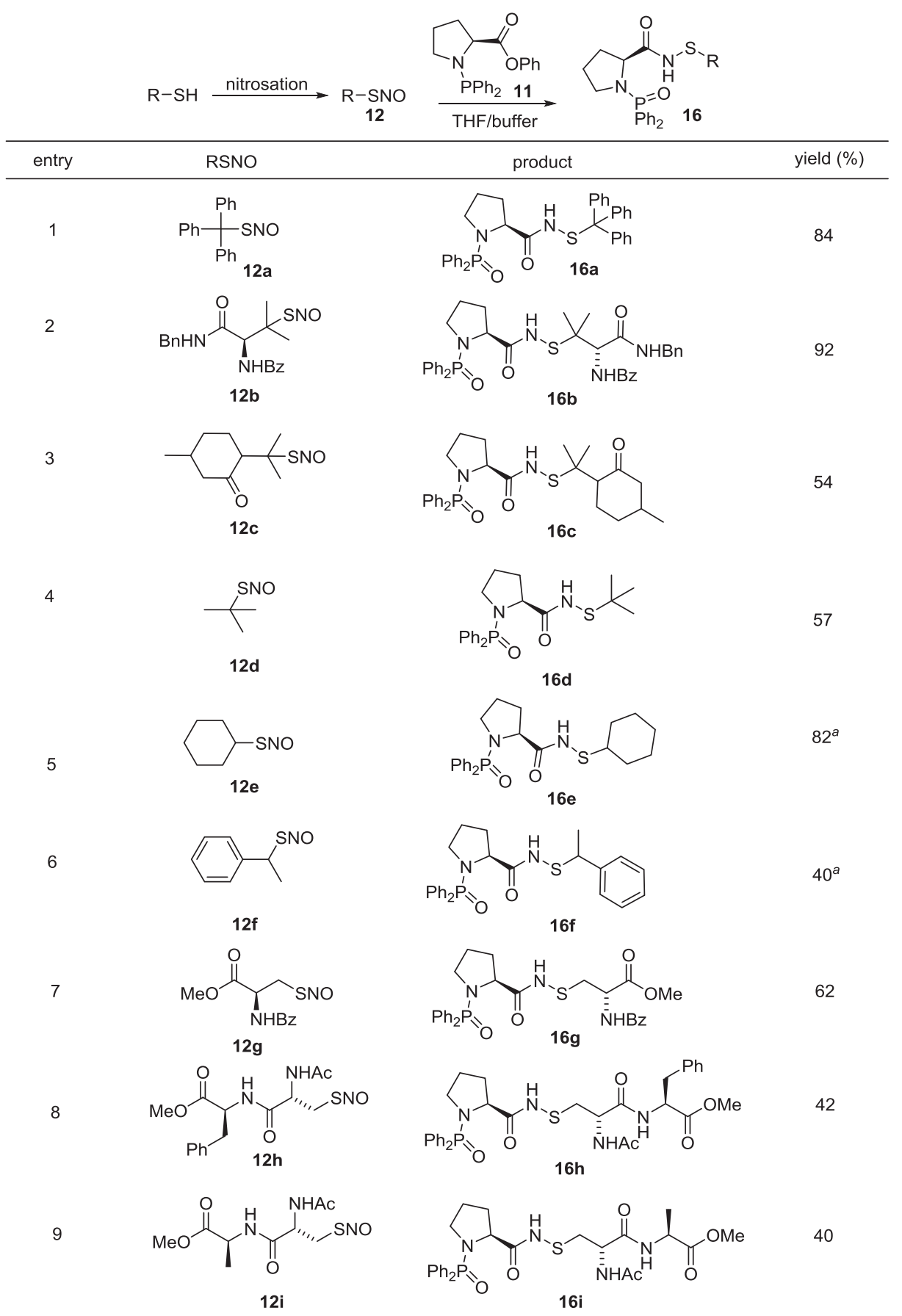

aYields detemined by NMR analysis.

phosphine oxide by-product. Nevertheless, the formation of the products was clearly confirmed by NMR and mass spectroscopy analysis. These results demonstrated that RSH can be readily converted to proline-based sulfenamides by this two-step SNO-ligation process. Although phosphoramidite $\mathbf{9}$ did not show good reactivity to convert RSNO to the desired sulfenamides, we did observe some unique reactivity of $\mathbf{9}$. As shown in Scheme 3, the treatment of a cysteine SNO derivative $\mathbf{1 2 g}$ with 9 gave dehydroalanine 18 in a modest yield (37\%) together with the disulfide product $(46 \%)$.
These results indicated that the reaction indeed proceeded to form the thioazaylide intermediate 17. Presumably, the methyl ester was not reactive enough. Hence, the acyl transfer was slow and nonproductive. Instead, the azaylide underwent an intramolecular $\beta$-elimination on the cysteine substrate to form dehydroalanine 18.

In the proline-based sulfenamide products, the diphenylphosphoryl moiety could be considered as the protecting group of proline. We expected it could be removed under acidic 


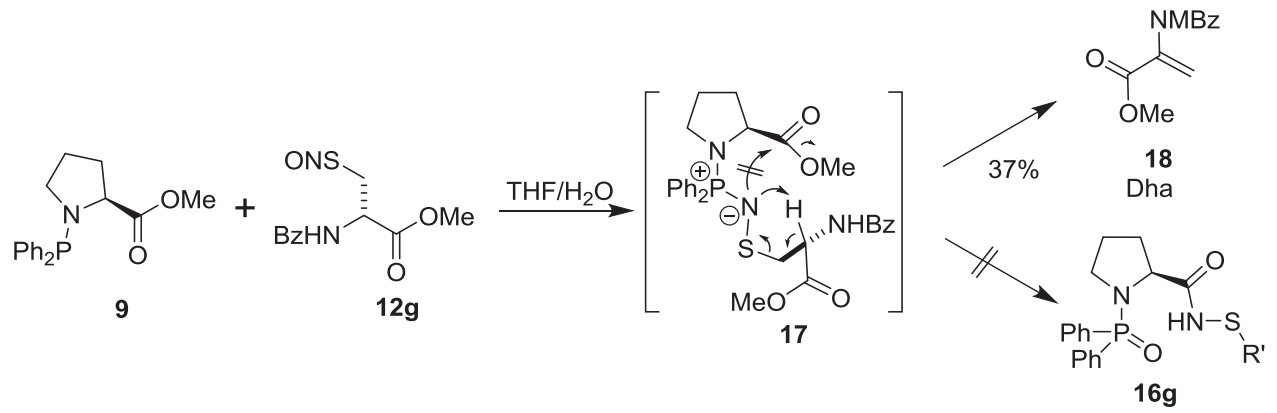

Scheme 3 The reaction between 9 and $\mathbf{1 2 g}$.<smiles>COC(=O)[C@H](N)CSNC(=O)[C@@H]1CCCN1[PH](=O)Pc1ccccc1</smiles>

$16 \mathrm{~g}$<smiles>COC(=O)[C@H](CSNC(=O)[C@@H]1CCCN1)C(=O)OCc1ccccc1</smiles>

19

Scheme 4 Deprotection of $16 \mathrm{~g}$.

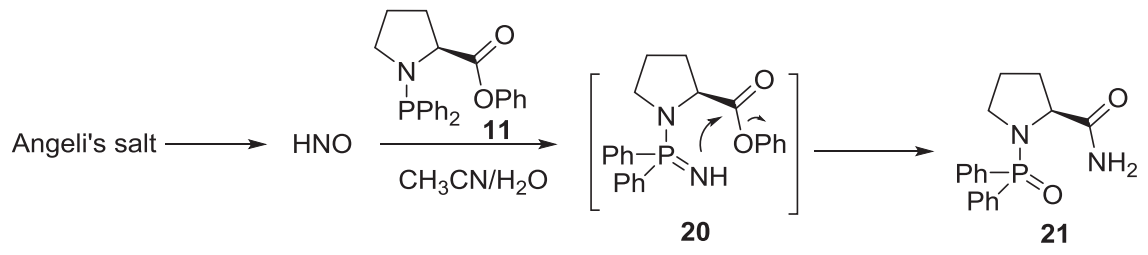

Scheme 5 The reaction between 11 and HNO.

conditions. ${ }^{42}$ We then tested the deprotection of $\mathbf{1 6 g}$ (Scheme 4). Indeed, a cleavage cocktail of $10 \%$ water in TFA provided sulfenamide 19 almost quantitatively. This result revealed that the $-\mathrm{S}-\mathrm{N}$ - bond on the sulfenamide compounds was quite stable under acidic conditions. Given the efficiency of this protocol, it can find applications in making water-soluble proline-based sulfenamides.

HNO is the one-electron reduced/protonated form of nitric oxide. It shows distinct physiology and pharmacology from nitric oxide. ${ }^{43}$ The reductive ligation was also found to be effective for HNO. ${ }^{44}$ Several specific fluorescent probes for $\mathrm{HNO}$ detection have been developed based on the triarylphosphine template. ${ }^{45-47}$ We next wondered whether the proline-based phosphoramidite would work for $\mathrm{HNO}$ and tested the reaction between $\mathbf{1 1}$ and HNO (generated from Angeli's salt). As shown in Scheme 5, the reaction proved to be fast and efficient. The desired ligation product 21 was obtained in a comparable yield (50\%) as the one obtained from triarylphosphines, ${ }^{44}$ suggesting the proline-based phosphoramidite may be useful for the design of novel HNO sensors.

\section{CONCLUSIONS}

In summary, we have developed a reductive ligation of $S$-nitrosothiols using $N$-diphenylphosphine proline ester substrates. This reaction was found to be effective for all small-molecule $S$-nitrosothiols (primary, secondary and tertiary), as well as for HNO. The ligation products bear a removable phosphine oxide moiety on the proline residue. In conjugation with the facile preparation of RSNOs from the corresponding $\mathrm{RSH}$, this novel method provides a unique way to prepare proline-based bioactive sulfenamides from simple thiol starting materials.

\section{METHODS}

\section{Instrumentation}

${ }^{1} \mathrm{H}$ NMR spectra, ${ }^{13} \mathrm{C}$ NMR and ${ }^{31} \mathrm{P}$ NMR were recorded at $300 \mathrm{MHz}$ (VX 300, Varian, Palo Alto, CA, USA) and are reported in p.p.m. on the $\delta$ scale relative to residual $\mathrm{CHCl}_{3}\left(\delta 7.25\right.$ for ${ }^{1} \mathrm{H}$ and $\delta 77.0$ for ${ }^{13} \mathrm{C}$ ). These experiments were performed at room temperature. Mass spectra were recorded using an electrospray ionization mass spectrometry (ESI, Thermo Finnigan LCQ Advantage, San Jose, CA, USA) or MALDI-TOF (matrix-assisted laser desorption ionization time-of-flight) mass spectrometry. Mass data were reported in units of $m / z$ for $[\mathrm{M}+\mathrm{H}]^{+}$or $[\mathrm{M}+\mathrm{Na}]^{+}$.

\section{Preparation of proline-based phosphoramidites}

To a solution of the proline ester $\mathrm{HCl}$ salt in $\mathrm{CH}_{2} \mathrm{Cl}_{2}$ (total $c=0.30 \mathrm{M}$ ) was added freshly distilled 2.5 equivalent of triethylamine followed by $\mathrm{PPh}_{2} \mathrm{Cl}$ (1 equivalent) at $0{ }^{\circ} \mathrm{C}$. The mixture was slowly warmed to room temperature and stirred for 3-4h. Upon completion (monitored by TLC), the reaction mixture was filtered to remove the triethylammonium salt. The filtrate was concentrated and diluted with EtOAc, and washed with satd $\mathrm{NaHCO}_{3}$, water and brine. The organic layer was dried with anhydrous $\mathrm{MgSO}_{4}$, filtered and concentrated. The crude product was purified by flash column chromatography (with preneutralized silica gel by $3 \%$ triethylamine in hexanes).

Compound 9: ${ }^{1} \mathrm{H}$ NMR $\left(300 \mathrm{MHz}, \mathrm{CDCl}_{3}\right) \delta$ 7.59-7.27 (m, 10H), 4.22 (ddd, $J=8.8,6.0,3.5 \mathrm{~Hz}, 1 \mathrm{H}), 3.63(\mathrm{~s}, 3 \mathrm{H}), 3.17-3.06(\mathrm{~m}, 1 \mathrm{H}), 2.88(\mathrm{~m}, 1 \mathrm{H}), 2.22-$ $1.70(\mathrm{~m}, 4 \mathrm{H}) ;{ }^{31} \mathrm{P}$ NMR $\left(122 \mathrm{MHz}, \mathrm{CDCl}_{3}\right) \delta 49.31 ;{ }^{13} \mathrm{C} \mathrm{NMR}(75 \mathrm{MHz}$, $\left.\mathrm{CDCl}_{3}\right) \delta 176.00,138.93,132.88,132.61,132.23,131.98,128.90,128.40,128.33$, $128.27,128.19,65.28,64.87,51.96,47.64,47.57,31.80,31.71,25.90$; MS (ESI) $\mathrm{m} / z$ calcd for $\mathrm{C}_{18} \mathrm{H}_{21} \mathrm{NO}_{2} \mathrm{P}[\mathrm{M}+\mathrm{H}]^{+} 314.1$, found 314.1.

Compound 10: ${ }^{1} \mathrm{H}$ NMR $\left(300 \mathrm{MHz}, \mathrm{CDCl}_{3}\right) \delta 7.49$ (ddd, $J=8.1,5.4,1.5 \mathrm{~Hz}$, $2 \mathrm{H}), 7.39-7.28(\mathrm{~m}, 8 \mathrm{H}), 4.07$ (ddd, $J=8.6,6.6,3.4 \mathrm{~Hz}, 1 \mathrm{H}), 3.17-3.06(\mathrm{~m}, 1 \mathrm{H})$, $2.83(\mathrm{dtd}, J=9.2,6.8,2.7 \mathrm{~Hz}, 1 \mathrm{H}), 2.07(\mathrm{dq}, J=12.0,8.3 \mathrm{~Hz}, 1 \mathrm{H}), 1.99-1.82$ $(\mathrm{m}, 2 \mathrm{H}), 1.76-1.64(\mathrm{~m}, 1 \mathrm{H}), 1.40(\mathrm{~s}, 9 \mathrm{H}) .{ }^{13} \mathrm{C} \mathrm{NMR}(75 \mathrm{MHz}, \mathrm{CDCl} 3)$ $\delta 174.76,139.27,132.93,132.66,132.26,132.01,128.81,128.38,128.29,128.19$, 
$128.12,80.76,66.37,65.95,47.72,47.65,31.83,31.74,28.24,25.87 .{ }^{31} \mathrm{P}$ NMR $\left(122 \mathrm{MHz}, \mathrm{CDCl}_{3}\right) \delta 49.64$.

Compound 11: ${ }^{1} \mathrm{H}$ NMR $\left(300 \mathrm{MHz}, \mathrm{CDCl}_{3}\right) \delta 7.59-7.15(\mathrm{~m}, 13 \mathrm{H})$, 7.07-6.95 (m, 2H), 4.47 (ddd, $J=8.3,6.3,3.8 \mathrm{~Hz}, 1 \mathrm{H}$ ), 3.21 (dddd, $J=8.7$, 7.5, 5.1, $1.0 \mathrm{~Hz}, 1 \mathrm{H}), 2.95(\mathrm{dtd}, J=9.4,7.0,2.4 \mathrm{~Hz}, 1 \mathrm{H}), 2.36-2.14(\mathrm{~m}, 2 \mathrm{H})$, 2.09-1.93 (m, $1 \mathrm{H}), 1.83(\mathrm{~m}, 1 \mathrm{H}) ;{ }^{31} \mathrm{P}$ NMR $\left(122 \mathrm{MHz}, \mathrm{CDCl}_{3}\right) \delta 49.99$; ${ }^{13} \mathrm{C}$ NMR $\left(75 \mathrm{MHz}, \mathrm{CDCl}_{3}\right) \delta 174.0,150.9,138.8,138.7,138.6,132.9,132.7$, $132.2,131.9,129.5,129.0,128.5,128.3,128.2,125.9,121.6,65.6,65.1,47.8$, 47.7, 32.0, 31.9, 25.9; MS (ESI) $\mathrm{m} / z$ calcd for $\mathrm{C}_{23} \mathrm{H}_{23} \mathrm{NO}_{2} \mathrm{P}[\mathrm{M}+\mathrm{H}]^{+} 376.1$, found 376.2

\section{Preparation of $S$-nitrosothiols}

The thiol starting material (RSH, $0.2 \mathrm{mmol}$ ) was dissolved in $1 \mathrm{ml}$ of $\mathrm{MeOH}$ followed by the addition of $1 \mathrm{~N} \mathrm{HCl}(1 \mathrm{ml})$ at room temperature. To this solution was then added freshly prepared $1 \mathrm{~N} \mathrm{NaNO}_{2}(1 \mathrm{ml})$ in water in dark (total $c=0.07 \mathrm{M}$ ). The color of the reaction was immediately turned to red (for primary and secondary RSNO) or green (for tertiary RSNO). The mixture was stirred for 10-15 min at room temperature. Upon completion (monitored by TLC), the RSNO product was directly extracted with cold diethyl ether $(1 \mathrm{ml} \times 3)$ in dark. The organic layers were collected and dried. The solvent was removed to provide the RSNO product that was then used for the ligation reaction without further purification.

\section{General reductive ligation procedure}

To the freshly prepared RSNO product was added a solution of 2 equivalent of 11 in 3:1 THF-aqueous buffer ( $\mathrm{pH}$ 7.4, degassed by bubbling with argon). The final concentration was $\sim 0.1 \mathrm{M}$. The reaction was monitored by TLC and it was usually completed within $15-30 \mathrm{~min}$ at room temperature. The reaction mixture was extracted with ethyl acetate. The combined organic layers were washed with water and brine, dried by anhydrous $\mathrm{Na}_{2} \mathrm{SO}_{4}$ and concentrated. The crude product was purified by flash column chromatography.

Thioazaylide 15: ${ }^{1} \mathrm{H}$ NMR $\left(300 \mathrm{MHz}, \mathrm{CDCl}_{3}\right) \delta$ 7.81-7.55 (m, $\left.4 \mathrm{H}\right)$, 7.47-7.17 (m, 23H), 6.94-6.80 (m, 3H), $4.35(\mathrm{td}, J=8.8,3.5 \mathrm{~Hz}, 1 \mathrm{H}), 3.14$ (qd, $J=6.5,2.8 \mathrm{~Hz}, 2 \mathrm{H}), 2.30(\mathrm{~m}, 1 \mathrm{H}), 2.13-1.99(\mathrm{~m}, 1 \mathrm{H}), 1.99-1.78(\mathrm{~m}, 2 \mathrm{H})$; ${ }^{31} \mathrm{P}\left(122 \mathrm{MHz}, \mathrm{CDCl}_{3}\right) \delta 18.3$; FT-IR (thin film) 3025.4, 2985.3, 1728.8 (strong, $\mathrm{C}=\mathrm{O}$, carbonyl group), $1601.3,1450.0,1372.3,1268.0,1247.1,1070.2 \mathrm{~cm}^{-1}$ MS (ESI) $m / z$ calcd for $\mathrm{C}_{42} \mathrm{H}_{37} \mathrm{~N}_{2} \mathrm{NaO}_{2} \mathrm{PS}[\mathrm{M}+\mathrm{Na}]^{+} 687.2$, found 687.1.

Compound 16a: ${ }^{1} \mathrm{H}$ NMR $\left(300 \mathrm{MHz}, \mathrm{CDCl}_{3}\right) \delta 7.81(\mathrm{~s}, 1 \mathrm{H}), 7.76-7.24$ $(\mathrm{m}, 25 \mathrm{H}), 3.95(\mathrm{dt}, J=9.2,5.5 \mathrm{~Hz}, 1 \mathrm{H}), 3.01(\mathrm{p}, J=8.1 \mathrm{~Hz}, 1 \mathrm{H}), 2.66-2.51$ $(\mathrm{m}, 1 \mathrm{H}), 1.92(\mathrm{~m}, 2 \mathrm{H}), 1.68-1.61(\mathrm{~m}, 1 \mathrm{H}), 1.34-1.26(\mathrm{~m}, 1 \mathrm{H}) ;{ }^{31} \mathrm{P}(122 \mathrm{MHz}$, $\left.\mathrm{CDCl}_{3}\right) \delta 30.2 ;{ }^{13} \mathrm{C} \mathrm{NMR}\left(75 \mathrm{MHz}, \mathrm{CDCl}_{3}\right) \delta 178.99,150.71,145.25,133.24$, $133.12,132.87,132.74,132.01,131.59,130.54,130.04,129.81,129.51,128.59$, $128.41,128.22,128.15,127.56,126.42,126.00,121.61,120.73,115.57,63.10$, $60.11,49.03,32.18,25.76$; MS (ESI) $\mathrm{m} / z$ calcd for $\mathrm{C}_{36} \mathrm{H}_{33} \mathrm{~N}_{2} \mathrm{NaO}_{2} \mathrm{PS}[\mathrm{M}+\mathrm{Na}]^{+}$ 611.2, found 611.1.

Compound 16b: ${ }^{1} \mathrm{H}$ NMR $\left(300 \mathrm{MHz}, \mathrm{CDCl}_{3}\right) \delta 9.55(\mathrm{t}, 5.6 \mathrm{~Hz}, 1 \mathrm{H}, \mathrm{NH})$ ), $9.12(\mathrm{~d}, J=9.5 \mathrm{~Hz}, 1 \mathrm{H}, \mathrm{NH})), 8.88(\mathrm{~s}, 1 \mathrm{H}, \mathrm{NH}), 8.12-6.82(\mathrm{~m}, 20 \mathrm{H}), \overline{5} .18$ $(\mathrm{d}, J=9.0 \mathrm{~Hz}, 1 \mathrm{H}), 4.46-4 . \overline{33}(\mathrm{~m}, 2 \mathrm{H}), 4.20-4 . \overline{2}(\mathrm{~m}, 1 \mathrm{H}), 3.44(\mathrm{~m}, 1 \mathrm{H}), 3.31$ $(\mathrm{m}, 1 \mathrm{H}), 2.39(\mathrm{~m}, 1 \mathrm{H}), 2.17(\mathrm{~m}, 3 \mathrm{H}), 1.36(\mathrm{~d}, J=10.8 \mathrm{~Hz}, 6 \mathrm{H}) ;{ }^{31} \mathrm{P}(122 \mathrm{MHz}$, $\left.\mathrm{CDCl}_{3}\right) \delta 30.2 ;{ }^{13} \mathrm{C} \mathrm{NMR}\left(75 \mathrm{MHz}, \mathrm{CDCl}_{3}\right) \delta 173.13,169.58,168.43,157.53$, $150.52,133.19,133.06,133.03,132.99,132.53,132.39,132.21,132.06,131.05$, $130.90,130.49,129.75,129.68,129.62,129.33,129.16,129.01,128.93,128.85$, $128.61,128.23,128.12,127.42,126.29,121.54,121.42,119.59,115.85,62.20$, $60.24, \quad 55.76, \quad 47.78, \quad 43.79, \quad 32.61, \quad 25.91$; MS (ESI) $\mathrm{m} / \mathrm{z}$ calcd for $\mathrm{C}_{36} \mathrm{H}_{39} \mathrm{~N}_{4} \mathrm{NaO}_{4} \mathrm{PS}[\mathrm{M}+\mathrm{Na}]^{+}$677.2, found 677.1.

Compound 16c: ${ }^{1} \mathrm{H}$ NMR $\left(400 \mathrm{MHz}, \mathrm{CDCl}_{3}\right) \delta 8.72(\mathrm{~s}, 1 \mathrm{H}), 7.82(\mathrm{~m}, 4 \mathrm{H})$, 7.58-7.40 (m, 6H), $4.18(\mathrm{t}, J=7.5 \mathrm{~Hz}, 1 \mathrm{H}), 3.19(\mathrm{dd}, J=12.2,6.0 \mathrm{~Hz}, 2 \mathrm{H})$, 2.65-2.25 (m, 5H), $2.03(\mathrm{t}, J=11.8 \mathrm{~Hz}, 2 \mathrm{H}), 1.90(\mathrm{dd}, J=13.6,10.1 \mathrm{~Hz}, 5 \mathrm{H})$, $1.42(\mathrm{~d}, J=7.3 \mathrm{~Hz}, 3 \mathrm{H}), 1.25(\mathrm{~d}, J=8.4 \mathrm{~Hz}, 3 \mathrm{H}), 1.00(\mathrm{~d}, J=6.0 \mathrm{~Hz}, 2.5 \mathrm{H})$, $0.93(\mathrm{~d}, J=7.1 \mathrm{~Hz}, 0.5 \mathrm{H}) ;{ }^{31} \mathrm{P}\left(122 \mathrm{MHz}, \mathrm{CDCl}_{3}\right) \delta 28.70 ;{ }^{13} \mathrm{C} \quad \mathrm{NMR}$ $\left(101 \mathrm{MHz}, \mathrm{CDCl}_{3}\right) \delta 211.43,175.80,132.34,132.25,132.15,131.96,131.86$, $128.89,128.79,128.66,77.32,77.00,76.68,62.25,57.80,52.67,52.24,48.20$, $36.73,34.47,31.11,31.05,30.90,29.74,25.64,25.16,25.10,22.28,22.19$; MS (Maldi) $\mathrm{m} / z$ calcd for $\mathrm{C}_{27} \mathrm{H}_{36} \mathrm{~N}_{2} \mathrm{O}_{3} \mathrm{PS}[\mathrm{M}+\mathrm{H}]^{+} 499.2178$, found 499.2201 .

Compound 16d: ${ }^{1} \mathrm{H}$ NMR $\left(300 \mathrm{MHz}, \mathrm{CDCl}_{3}\right) \delta 8.64$ (br-s, $\left.1 \mathrm{H}, \mathrm{NH}\right)$,

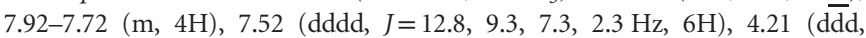

$J=8.5,6.5,2.2 \mathrm{~Hz}, 1 \mathrm{H}), 3.20(\mathrm{~m}, 2 \mathrm{H}), 2.43(\mathrm{dd}, J=12.7,5.9 \mathrm{~Hz}, 1 \mathrm{H})$, 2.13-2.02 (m, 1H), $1.91(\mathrm{~m}, 2 \mathrm{H}), 1.28(\mathrm{~s}, 9 \mathrm{H}) ;{ }^{31} \mathrm{P}\left(122 \mathrm{MHz}, \mathrm{CDCl}_{3}\right)$ $\delta 30.0 ;{ }^{13} \mathrm{C} \mathrm{NMR}\left(75 \mathrm{MHz}, \mathrm{CDCl}_{3}\right) \delta 175.98,132.67,132.52,132.39,132.32$, $132.19,129.65,129.25,129.13,129.08,128.97,121.40,62.58,48.91,48.35$, $31.49,28.92,25.42,25.33$; MS (Maldi) $\mathrm{m} / z$ calcd for $\mathrm{C}_{21} \mathrm{H}_{28} \mathrm{~N}_{2} \mathrm{O}_{2} \mathrm{PS}[\mathrm{M}+\mathrm{H}]^{+}$ 403.1609, found 403.1604 .

Compound 16g: ${ }^{1} \mathrm{H}$ NMR $\left(300 \mathrm{MHz}, \mathrm{CDCl}_{3}\right) \quad \delta \quad 8.97 \quad(\mathrm{~s}, \quad 1 \mathrm{H}), \quad 8.30$ $(\mathrm{d}, J=8.3 \mathrm{~Hz}, 1 \mathrm{H}), \quad 8.09-7.97(\mathrm{~m}, 2 \mathrm{H}), \quad 7.90-7.67(\mathrm{~m}, 4 \mathrm{H}), \quad 7.62-7.36$ $(\mathrm{m}, 9 \mathrm{H}), 5.02(\mathrm{ddd}, J=8.3,6.3,4.3 \mathrm{~Hz}, 1 \mathrm{H}), 4.27-4.02(\mathrm{~m}, 1 \mathrm{H}), 3.74$ (s, 3H), $3.32(\mathrm{dd}, J=14.8,6.4 \mathrm{~Hz}, 1 \mathrm{H}), 3.04(\mathrm{~m}, 3 \mathrm{H}), 2.35-2.17(\mathrm{~m}, 1 \mathrm{H})$, $2.01-1.84(\mathrm{~m}, 1 \mathrm{H}), 1.72(\mathrm{~m}, 2 \mathrm{H}) ;{ }^{31} \mathrm{P}\left(122 \mathrm{MHz}, \mathrm{CDCl}_{3}\right) \delta 30.1 ;{ }^{13} \mathrm{C} \mathrm{NMR}$ $\left(75 \mathrm{MHz}, \mathrm{CDCl}_{3}\right) \delta 176.84,171.32,167.73,133.84,132.72,132.50,132.37$, $132.21,132.07,131.97,129.21,129.10,128.93,128.65,127.91,61.90,52.96$, $51.28,48.72,42.00,30.95,25.32$; MS (ESI) $\mathrm{m} / z$ calcd for $\mathrm{C}_{28} \mathrm{H}_{30} \mathrm{~N}_{3} \mathrm{NaO}_{5} \mathrm{PS}$ $[\mathrm{M}+\mathrm{Na}]^{+}$574.3, found 574.3 .

Compound 16h: ${ }^{1} \mathrm{H}$ NMR $\left(300 \mathrm{MHz}, \mathrm{CDCl}_{3}\right) \delta 8.92(\mathrm{~d}, J=7.6 \mathrm{~Hz}, 1 \mathrm{H}), 8.44$ (s, $1 \mathrm{H}), 7.75$ (tdd, $J=12.0,8.3,1.4 \mathrm{~Hz}, 4 \mathrm{H}), 7.67-7.38(\mathrm{~m}, 6 \mathrm{H}), 7.01$ (d, $J=7.2 \mathrm{~Hz}, 3 \mathrm{H}), 6.97-6.85(\mathrm{~m}, 3 \mathrm{H}), 5.38(\mathrm{td}, J=9.5,3.2 \mathrm{~Hz}, 1 \mathrm{H}), 4.63$ $(\mathrm{td}, J=8.3,5.0 \mathrm{~Hz}, 1 \mathrm{H}), 4.07(\mathrm{td}, J=7.0,5.0 \mathrm{~Hz}, 1 \mathrm{H}), 3.67(\mathrm{~s}, 3 \mathrm{H}), 3.42$ (m, 1H), 3.27-2.99 (m, 3H), $2.84(\mathrm{dd}, J=13.9,8.9 \mathrm{~Hz}, 1 \mathrm{H}), 2.49$ (dd, $J=14.1$, $9.9 \mathrm{~Hz}, 1 \mathrm{H}), 2.23-2.10(\mathrm{~m}, 2 \mathrm{H}), 2.08(\mathrm{~s}, 3 \mathrm{H}), 2.02-1.76(\mathrm{~m}, 3 \mathrm{H}) ;{ }^{31} \mathrm{P}$ $\left(122 \mathrm{MHz}, \mathrm{CDCl}_{3}\right) \delta 30.4 ;{ }^{13} \mathrm{C} \mathrm{NMR}\left(75 \mathrm{MHz}, \mathrm{CDCl}_{3}\right) \delta 177.22,171.75$, $171.46,171.04,136.73,132.65,132.52,132.33,132.19,129.31,129.20,129.10$, $129.04,128.93,128.41,126.81,62.41,54.36,52.55,49.93,43.67,37.95,25.70$, 23.40; MS (ESI) $\mathrm{m} / z$ calcd for $\mathrm{C}_{32} \mathrm{H}_{37} \mathrm{~N}_{4} \mathrm{NaO}_{6} \mathrm{PS}[\mathrm{M}+\mathrm{Na}]^{+} 659.2$, found 659.3 .

Compound 16i: ${ }^{1} \mathrm{H}$ NMR $\left(300 \mathrm{MHz}, \mathrm{CDCl}_{3}\right) \delta 8.21(\mathrm{~d}, J=7.4 \mathrm{~Hz}, 1 \mathrm{H}), 7.80$ $(\mathrm{m}, 5 \mathrm{H}), 7.45(\mathrm{~m}, 6 \mathrm{H}), 7.19(\mathrm{~d}, J=8.1 \mathrm{~Hz}, 1 \mathrm{H}), 4.93(\mathrm{~m}, 1 \mathrm{H}), 4.75(\mathrm{~m}, 1 \mathrm{H})$, $4.50(\mathrm{~m}, 1 \mathrm{H}), 4.01$ (ddd, $J=8.2,5.9,2.3 \mathrm{~Hz}, 2 \mathrm{H}), 3.75-3.57(\mathrm{~m}, 3 \mathrm{H}), 3.29-3.11$ $(\mathrm{m}, 2 \mathrm{H}), 2.40-2.24(\mathrm{~m}, 1 \mathrm{H}), 2.10-2.03(\mathrm{~m}, 1 \mathrm{H}), 1.98(\mathrm{~m}, 3 \mathrm{H}), 1.92-1.81$ $(\mathrm{m}, 4 \mathrm{H}), 1.47-1.29(\mathrm{~m}, 3 \mathrm{H}) ;{ }^{31} \mathrm{P}\left(122 \mathrm{MHz}, \mathrm{CDCl}_{3}\right) \delta 30.4 ;{ }^{13} \mathrm{C} \mathrm{NMR}$ $\left(75 \mathrm{MHz}, \mathrm{CDCl}_{3}\right) \delta 171.22,170.56,170.56,170.08,132.61,132.57,132.54$, $132.51,132.42,132.37,132.30,132.24,131.76,131.52,130.05,129.84,129.19$, $129.10,129.03,128.93,62.06,62.02,53.70,52.63,48.47,48.06,48.01,42.04$, $31.27,31.19,25.26,25.17,23.24,17.97,17.57$; MS (ESI) $\mathrm{m} / z$ calcd for $\mathrm{C}_{26} \mathrm{H}_{35} \mathrm{~N}_{4} \mathrm{NaO}_{5} \mathrm{PS}[\mathrm{M}+\mathrm{Na}]^{+} 569.2$, found 569.3 .

Dehydroalanine (Dha) 18: ${ }^{1} \mathrm{H}$ NMR $\left(300 \mathrm{MHz}, \mathrm{CDCl}_{3}\right) \delta 8.54$ (br, $\left.1 \mathrm{H}\right), 7.85-$ $7.81(\mathrm{~m}, 2 \mathrm{H}), 7.57-7.43(\mathrm{~m}, 3 \mathrm{H}), 6.79(\mathrm{~s}, 1 \mathrm{H}), 5.98(\mathrm{~d}, 1 \mathrm{H}, J=1.4 \mathrm{~Hz}), 3.88(\mathrm{~s}$, $3 \mathrm{H}) ;{ }^{13} \mathrm{C}$ NMR $\left(75 \mathrm{MHz}, \mathrm{CDCl}_{3}\right) \delta 166.0,165.0,134.4,132.3,131.2,129.0$, 127.1, 109.1, 53.3; MS (ESI) $\mathrm{m} / z$ calcd for $\mathrm{C}_{11} \mathrm{H}_{12} \mathrm{NO}_{3}[\mathrm{M}+\mathrm{H}]^{+}$206.1, found 206.2 .

\section{Deprotection of the diphenylphosphoryl group}

A sulfenamide product $(0.05 \mathrm{mmol})$ was treated with $1 \mathrm{ml}$ of cold $10 \%$ water in TFA at $0{ }^{\circ} \mathrm{C}$ (total $c=0.05 \mathrm{M}$ ). The resulted solution was stirred for $30 \mathrm{~min}$ at $0{ }^{\circ} \mathrm{C}$. Upon completion, the excess TFA was removed with hexanes as the cosolvent under vacuum. To the remaining mixture was added cold diethyl ether to solidify the proline-sulfenamide TFA salt. The solid product was further washed with cold diethyl ether $(2 \mathrm{ml} \times 5)$ and dried to provide the final product.

Compound 19: ${ }^{1} \mathrm{H}$ NMR $\left(300 \mathrm{MHz}, \mathrm{CDCl}_{3}\right) \delta 10.31$ (br-s, $\left.1 \mathrm{H}\right), 9.70$ (br-s, 1H), $9.09(\mathrm{~s}, 1 \mathrm{H}), 7.57-7.35(\mathrm{~m}, 5 \mathrm{H}), 4.91(\mathrm{~m}, 1 \mathrm{H}), 4.64(\mathrm{~m}, 1 \mathrm{H})$, $3.74(\mathrm{~s}, 3 \mathrm{H}), 3.38(\mathrm{~m}, 2 \mathrm{H}), 3.23(\mathrm{~m}, 2 \mathrm{H}), 2.32(\mathrm{~m}, 1 \mathrm{H}), 2.01(\mathrm{~m}, 3 \mathrm{H}) ;{ }^{13} \mathrm{C}$ NMR $\left(75 \mathrm{MHz}, \mathrm{CDCl}_{3}\right) \delta 172.40,171.21,168.61,132.96,128.96,128.70$, $127.61,60.54,53.21,51.71,47.05,40.52,29.64,24.59$; MS (Maldi) $\mathrm{m} / \mathrm{z}$ calcd for $\mathrm{C}_{16} \mathrm{H}_{22} \mathrm{~N}_{3} \mathrm{O}_{4} \mathrm{~S}[\mathrm{M}+\mathrm{H}]^{+}$352.1331, found 352.1320.

\section{The reaction between 11 and $\mathrm{HNO}$}

To an argon sparged mixture of acetonitrile and water was added 11 $(0.21 \mathrm{mmol})$, and to this stirring mixture was added freshly prepared Angeli's salt $\left(0.1 \mathrm{mmol}, \mathrm{Na}_{2} \mathrm{~N}_{2} \mathrm{O}_{3}\right)$. The resulting solution was allowed to stir until the reaction was completed (by TLC, or $20 \mathrm{~min}$ ). The product 21 (50\% yield) was isolated by extraction and flash column chromatography.

Compound 21: ${ }^{1} \mathrm{H}$ NMR $\left(300 \mathrm{MHz}, \mathrm{CDCl}_{3}\right) \delta 10.04$ (br-s, $\left.1 \mathrm{H}, \mathrm{NH}\right)$, 7.96-7.68 (m, 5H), 7.64-7.39 (m, 5H), 5.85 (br-s, 1H, NH), 4.03 (ddd, $J=8.1,5.7,2.3 \mathrm{~Hz}, 1 \mathrm{H}), 3.18(\mathrm{~m}, 2 \mathrm{H}), 2.46-2.18(\mathrm{~m}, 1 \mathrm{H}), 2.18-1.99(\mathrm{~m}, 1 \mathrm{H})$, 1.89 (ddt, $J=13.5,8.9,4.2 \mathrm{~Hz}, 2 \mathrm{H}) ;{ }^{31} \mathrm{P}\left(122 \mathrm{MHz}, \mathrm{CDCl}_{3}\right) \delta 28.6 ;{ }^{13} \mathrm{C} \mathrm{NMR}$ 
$\left(75 \mathrm{MHz}, \mathrm{CDCl}_{3}\right) \delta 176.32,132.66,132.62,132.59,132.55,132.44,132.41$, $132.31,132.28,131.65,131.39,129.92,129.71,129.22,129.13,129.05,128.96$, $62.12,62.08,60.45,48.07,48.02,31.29,31.21,25.27,25.18$; MS (ESI) $\mathrm{m} / z$ calcd for $\mathrm{C}_{17} \mathrm{H}_{19} \mathrm{~N}_{2} \mathrm{NaO}_{2} \mathrm{P}[\mathrm{M}+\mathrm{Na}]^{+}$337.1, found 337.1.

\section{CONFLICT OF INTEREST}

The authors declare no conflict of interest.

\section{ACKNOWLEDGEMENTS}

This work is supported by the NSF (0844931) and the NIH (R01GM088226).

1 Chaleff, R. S. \& Mauvais, C. J. Acetolactate synthase is the site of action of two sulfonylurea herbicides in higher plants. Science 224, 1443-1445 (1984).

2 Wang, J. G. et al. Structure-activity relationships for a new family of sulfonylurea herbicides. J. Comput. Aided Mol. Des. 19, 801-820 (2005).

3 Cooper, L. Z., Madoff, M. A. \& Weinstein, L. Absorption and excretion of sulfamethomidine, a "long-acting" sulfonamide. Antibiot. Chemother. 12, 618-629 (1962).

4 Shah, S. S., Rivera, G. \& Ashfaq, M. Recent advances in medicinal chemistry of sulfonamides. Rational design as anti-tumoral, anti-bacterial and antiinflammatory agents. Mini. Rev. Med. Chem. 13, 70-86 (2013).

5 Turos, E. et al. N-thiolated bicyclic and monocyclic $\beta$-lactams. Tetrahedron 56, 5571-5578 (2000)

6 O'Driscoll, M. et al. Studies on the antifungal properties of $N$-thiolated $\beta$-lactams. Bioorg. Med. Chem. 16, 7832-7837 (2008).

7 Prosen, K. R. et al. The impact of fatty acids on the antibacterial properties of $N$-thiolated $\beta$-lactams. Bioorg. Med. Chem. Lett. 21, 5293-5295 (2011).

8 Revell, K. D., Heldreth, B., Long, T. E., Jang, S. \& Turos, E. $N$-thiolated $\beta$-lactams: studies on the mode of action and identification of a primary cellular target in Staphylococcus aureus. Bioorg. Med. Chem. 15, 2453-2467 (2007).

9 Heldreth, B. et al. $N$-Thiolated $\beta$-lactam antibacterials: effects of the $N$-organothio substituent on anti-MRSA activity. Bioorg. Med. Chem. 14, 3775-3784 (2006).

10 Turos, E. et al. $N$-Thiolated $\beta$-lactams: a new family of anti-Bacillus agents. Bioorg. Med. Chem. Lett. 16, 2084-2090 (2006).

11 Turos, E. et al. $N$-thiolated $\beta$-lactams: novel antibacterial agents for methicillin-resistant Staphylococcus aureus. Bioorg. Med. Chem. Lett. 12, 2229-2231 (2002).

12 Mishra, R. K. et al. $N$-thiolated 2-oxazolidinones: a new family of antibacterial agents for methicillin-resistant Staphylococcus aureus and Bacillus anthracis. Bioorg. Med. Chem. Lett. 16, 2081-2083 (2006).

13 Shang, J. L. et al. Synthesis and evaluation of novel sulfenamides as novel anti methicillin-resistant Staphylococcus aureus agents. Bioorg. Med. Chem. Lett. 23, 724-727 (2013)

14 Crasto, C. F. et al. Synthesis and activity of analogues of the isoleucyl tRNA synthetase inhibitor SB-203207. Bioorg. Med. Chem. 11, 2687-2694 (2003).

15 Stefanska, A. L., Cassels, R., Ready, S. J. \& Warr, S. R. SB-203207 and SB-203208, two novel isoleucyl tRNA synthetase inhibitors from a Streptomyces sp. I. Fermentation, isolation and properties. J. Antibiot. (Tokyo) 53, 357-363 (2000).

16 Huang, S. et al. 1,2,4-Triazolylaminoarylsulfonamide derivatives as antiproliferative agents, their preparation, pharmaceutical compositions, and use in therapy, W02006042215A1 (2006).

17 Koval, I. V. Progress in the chemistry of perhalomethanesulphenyl halides. Russ. Chem. Rev. 60, 830-849 (1991).

18 Koval, I. V. Sylfenyl chlorides in organic synthesis. Russ. Chem. Rev. 64, 731-751 (1995)

19 Koval, I. V. Thiols as synthons. Russ. Chem. Rev. 62, 769-786 (1993)

20 Davis, F. A. et al. Chemistry of the sulfur-nitrogen bond. VI. Convenient one-step synthesis of sulfenimines (S-aryl thiooximes). J. Org. Chem. 38, 2809-2813 (1973).

21 Davis, F. A. et al. Chemistry of the sulfur-nitrogen bond. 12. Metal-assisted synthesis of sulfenamide derivatives from aliphatic and aromatic disulfides. J. Org. Chem. 42, 967-972 (1977)
22 Petrov, K. A., Rudnev, G. V. \& Sorokin, V. D. Sulphenamides and their derivatives. Russ. Chem. Rev. 59, 832-843 (1990).

23 Mueller, W. H. \& Butler, P. E. $\beta$-Chloroalkylsulfenamides. Addition of dimethylaminosulfenyl chloride to unsaturated hydrocarbons. J. Org. Chem. 33, 2111-2113 (1968).

24 Kühle, E. One hundred years of sulfenic acid chemistry IIb. Substitution and cyclization reactions of sulfenyl halides. Synthesis 1971, 617-638 (1971).

25 Koval, I. V. Progress in the chemistry of sulphilimines. Russ. Chem. Rev. 59, 819-831 (1990).

26 Pel'kis, N. P. \& Levchenko, E. S. Interation of areniminosulfinic acid-derivatives with thiophenols. Zh. Org. Khim. 22, 387-391 (1986).

27 Torii, S., Tanaka, H. \& Ukida, M. Electrosynthesis of hetero-hetero atom bonds. 2. An efficient preparation of (2-benzothiazolyl)- and thiocarbamoylsulfenamides by electrolytic cross-coupling reaction of 2-mercaptobenzothiazole, bis(2-benzothiazolyl) disulfide, and/or bis(dialkylthiocarbamoyl) disulfides with various amines. J. Org. Chem. 43, 3223-3227 (1978)

28 Torii, S., Tanaka, H. \& Ukida, M. Electrosynthesis of hetero-hetero atom bonds. 3. Sodium bromide promoted electrolytic cross-coupling reaction of imides with disulfides. Convenient synthesis of $\mathrm{N}$-(cyclohexylthio)phthalimide, an important prevulcanization inhibitor. J. Org. Chem. 44, 1554-1557 (1979).

29 Davis, F. A., Friedman, A. J. \& Nadir, U. K. Chemistry of the sulfur-nitrogen bond. 14. Arenesulfenic acids from $\mathrm{N}$-alkylidenearenesulfinamides (sulfinimines). J. Am. Chem. Soc. 100, 2844-2852 (1978).

30 Haake, M., Gebbing, H. \& Benack, H. A simple method for the oxidation of sulfenamides to sulfinamides. Synthesis 1979, 97-97 (1979).

31 Zhang, Y. \& Hogg, N. S-Nitrosothiols: cellular formation and transport. Free Radical Biol. Med. 38, 831-838 (2005).

32 Lancaster, J. R. Jr. Protein cysteine thiol nitrosation: maker or marker of reactive nitrogen species-induced nonerythroid cellular signaling? Nitric Oxide 19, 68-72 (2008).

33 Smith, B. C. \& Marletta, M. A. Mechanisms of S-nitrosothiol formation and selectivity in nitric oxide signaling. Curr. Opin. Chem. Biol. 16, 498-506 (2012).

34 Wang, H. \& Xian, M. Fast reductive ligation of S-nitrosothiols. Angew. Chem. Int. Ed. 47, 6598-6601 (2008)

35 Zhang, J., Wang, H. \& Xian, M. Exploration of the "traceless" reductive ligation of S-nitrosothiols. Org. Lett. 11, 477-480 (2009).

36 Zhang, J. et al. Reductive ligation mediated one-step disulfide formation of S-nitrosothiols. Org. Lett. 12, 4208-4211 (2010).

37 Zhang, J., Wang, H. \& Xian, M. An unexpected Bis-ligation of S-nitrosothiols. J. Am. Chem. Soc. 131, 3854-3855 (2009).

38 Pan, J. \& Xian, M. Disulfide formation via sulfenamides. Chem. Commun. 47, 352-354 (2011)

39 Wang, H., Zhang, J. \& Xian, M. Facile formation of dehydroalanine from S-nitrosocysteines. J. Am. Chem. Soc. 131, 13238-13239 (2009).

40 Pan, J., Devarie-Baez, N. O. \& Xian, M. Facile amide formation via S-nitrosothioacids. Org. Lett. 13, 1092-1094 (2011).

41 Devarie-Baez, N. O., Zhang, D., Li, S., Whorton, A. R. \& Xian, M. Direct methods for detection of protein S-nitrosylation. Methods 62, 171-176 (2013).

42 Ramage, R., Hopton, D., Parrott, M. J., Kenner, G. W. \& Moore, G. A. Phosphinamides: a new class of amino protecting groups in peptide synthesis. J. Chem. Soc. Perkin Trans. 1, 1357-1370 (1984).

43 Fukuto, J. M. et al. The physiological chemistry and biological activity of nitroxyl (HNO): the neglected, misunderstood, and enigmatic nitrogen oxide. Chem. Res. Toxicol. 18, 790-801 (2005).

44 Reisz, J. A., Klorig, E. B., Wright, M. W. \& King, S. B. Reductive phosphine-mediated ligation of nitroxyl (HNO). Org. Lett. 11, 2719-2721 (2009).

45 Reisz, J. A., Zink, C. N. \& King, S. B. Rapid and selective nitroxyl (HNO) trapping by phosphines: kinetics and new aqueous ligations for HNO detection and quantitation. J. Am. Chem. Soc. 133, 11675-11685 (2011).

46 Miao, Z. et al. A selective phosphine-based fluorescent probe for nitroxyl in living cells. Bioorg. Med. Chem. Lett. 25, 16-19 (2015).

47 Kawai, K. et al. A reductant-resistant and metal-free fluorescent probe for nitroxyl applicable to living cells. J. Am. Chem. Soc. 135, 12690-12696 (2013). 